\title{
Knowledge on HIV testing indications and practice of requesting HIV tests by the intern medical officers in four tertiary care hospitals in Colombo; A descriptive cross sectional study.
}

\section{Premadasa PS ${ }^{1}$, Karawita DA ${ }^{2}$, Azran $A^{1}$, Widange WN $^{1}$, Dayaratne HGAK ${ }^{1}$, Ediriwickrama $\mathbf{N}^{3}$}

\begin{abstract}
Introduction: Sri Lanka being an HIV low prevalent country (prevalence $<0.1 \%$ in the general population), the ward clinicians have less clinical experience and exposure to HIV patients and HIV is considered lower down in their differential diagnosis. Therefore over the years the majority of adults who are detected with HIV in the ward setting were significantly immunocompromised at the time of diagnosis and in 2012 the mean CD4 count of the inward diagnosed HIV cases was 92.1 cells $/ \mu l^{1}$. Late diagnosis is associated with increased mortality, morbidity and impaired response to $\mathrm{ART}^{2}$. HIV testing in all patients presenting with signs and symptoms or medical conditions indicative of HIV infection facilitate early case detection; it can also encourage 'normalisation" of HIV testing.
\end{abstract}

Objectives: To assess the knowledge on indications to request an HIV test and practice of requesting HIV test by the intern medical officers (IMO) attached to 4 tertiary care hospitals in Colombo.

Methods: A descriptive cross sectional study was carried out among 100 out of 104 IMO attached to 4 tertiary care hospitals in Colombo, using a selfadministered questionnaire. Key outcomes included the knowledge on clinical indications for HIV testing, assessment of current practice of HIV testing, and knowledge and obstacles on routine testing procedure. Knowledge on clinical indications for HIV testing was assessed using the comprehensive list developed by the British HIV Association (BHIVA) which comprised of the conditions included in WHO clinical staging.

${ }^{1}$ Registrar in Venereology, National STD/AIDS Control Programme, ${ }^{2}$ Consultant Venereologist, Teaching Hospital Anuradhapura ${ }^{3}$, Registrar in Clinical Haematology National Hospital of Sri Lanka.

Corresponding author: Premadasa PS, Email: prageethsuranga@rocketmail.com 


\section{Keywords}

Clinical indications for HIV testing, knowledge.

\section{Introduction}

Even though Sri Lanka is currently experiencing a low level HIV epidemic, several socioeconomic and behavioural factors which can ignite an epidemic in near future, have been identified. Presence of a large youth population, increasing internal and external migration, concurrent sexual relationships among most at risk populations with low condom use are some such factors. ${ }^{3}$

As at end of December 2012, a cumulative total of 1649 HIV persons reported to the National STD/ AIDS Control Programme (NSACP), when the estimated number is $4200^{1}$. Therefore the healthcare system has not been able to detect more than 50\% of estimated HIV cases in Sri Lanka, irrespective of the efforts made by the NSACP with its multisectoral collaboration.

In 2012 a total of 146 cases of HIV were newly detected in Sri Lanka and from them 20 have been referred from ward setting for the diagnosis. In 2012, all the patients who were detected HIV positive while getting inward treatment has been detected with advanced (WHO stage 4) disease with a mean CD4 count of 92.1 cells $/ \mu 1^{1}$. Therefore most of the adults with HIV were significantly immunocompromised at the time of detection in the ward.

In the pre anti retroviral therapy (ART) era when only palliative care was given for persons living with HIV (PLHIV), routine HIV testing did not have any added benefits for the patients. However with the introduction of ART to Sri Lanka in 2004 the gloomy picture has been brightened dramatically and patients are now offered lifelong treatment that confers a similar life expectancy to that of a general population. The prognosis and further transmission are further improved with early detection of HIV before the onset of significant immunocompromisation.

Apart from that the availability of voluntary counselling and testing (VCT) or provider initiated testing for HIV has itself shown to decrease high risk sexual behavior, bringing the added benefit to public health of reduced onward transmission ${ }^{4}$. Therefore in this context, HIV screening tests are now offered routinely in various clinical settings in Sri Lanka for example; antenatal clinics, TB patients. Screening in these settings alone, however is not enough to detect all early cases of HIV and late diagnosis is the most important factor associated with HIV-related morbidity and mortality ${ }^{5}$. Patients should therefore be offered and encouraged to accept HIV testing in a wider range of settings than is currently the case. Patients with specific indicator conditions should be routinely recommended to have an HIV test by the clinicians; hence it can also encourage 'normalisation' of HIV testing. Late diagnosis of HIV infection has been associated with increased mortality and morbidity, impaired response to highly active anti retroviral therapy (HAART) and increased cost to healthcare services ${ }^{2}$

Many of the opportunities to diagnose and counsel individuals of health facilities are missed. Furthermore, most of the late diagnosed cases have had at least one factor that should have prompted health care providers to consider the need of HIV testing. In the case of persons presenting to health care facilities with signs and symptoms of illness that could be attributable to HIV, it is a basic responsibility of a health care provider to recommend HIV testing and counseling. To recommend HIV testing for the relevant populations, doctors' knowledge has to be adequate regarding the indications ${ }^{4}$

According to the WHO HIV testing recommendations, for all epidemic types, all adults and adolescents who present to health facilities with signs and symptoms or medical conditions that could indicate HIV infection should be screened. Furthermore, for the countries like Sri Lanka with low level epidemics universal screening is not recommended ${ }^{4}$

The pre-registration (internship) appointment is a continuing part of the medical education programme of a medical practitioner in Sri Lanka. The IMO are 
required to do first clerking of the patients who are admitted to the wards. Furthermore they are supposed to obtain a detailed history, carry out a complete examination and to order relevant investigations. IMO are required to see in- ward patients and order relevant investigations depending on the clinical findings. The main focus of this training is to gain practical experience in managing patients in the hospital setting before they are given the full license to practice medicine unsupervised within this country ${ }^{6}$

After one year of training which is comprised of two appointments of six months each, they are released as qualified medical officers to work in various clinical settings around the country. Current IMO have the future challenge of addressing the emerging health issues of the nation. Furthermore to turn back the future HIV epidemic in Sri Lanka their role becomes important by effective early detection of HIV related conditions and promoting HIV testing among suspected individuals.

\section{Justification}

Sri Lanka being an HIV low prevalent country (prevalence $<0.1 \%$ in the general population), the ward clinicians have less clinical experience and exposure to HIV patients and HIV is considered lower down in their differential diagnosis. Therefore over the years majority of the adults who are detected with HIV in the ward setting were significantly immunocompromised at the time of diagnosis and in 2012 the mean CD4 count of the inward diagnosed HIV cases was $92.1 \mathrm{cells} / \mu \mathrm{l}$. $^{1}$ Late diagnosis is associated with increased mortality, morbidity and impaired response to $\mathrm{ART}^{2}$. HIV testing in all patients presenting with signs and symptoms or medical conditions indicative of HIV infection facilitate early case detection; hence it can also encourage 'normalisation" of HIV testing. In the case of persons presenting to health care facilities with signs and symptoms of illness that could be attributable to HIV, it is a basic responsibility of a health care provider to recommend HIV testing and counseling. To recommend HIV testing for the relevant populations, doctors' knowledge has to be adequate regarding the indications. Therefore assessment of the knowledge and practice of doctors, who will be appointed soon for various healthcare institutions around the country, is important to make improved policy dispersal coupled with education that targets barriers for HIV testing.

\section{Methods}

A descriptive cross sectional study was carried out among 100 out of 104 Intern Medical officers attached to 4 tertiary care hospitals in Colombo, namely National hospital of Sri Lanka, De Soyza maternity hospital, Lady Ridgway hospital and Castle Street hospital for women. A pre tested self administered questionnaire was used to collect data. Key outcomes included the knowledge on clinical indications for HIV testing, assessment of current practice of HIV testing, Knowledge and obstacles on routine testing procedure. Knowledge on clinical indications for HIV testing was assessed using the comprehensive list developed by BHIVA which comprised of the conditions included in WHO clinical staging. Data were analyzed by using the statistical package for the social sciences version 16(SPSS v16). Ethical approval for the study was obtained from the ethical review committee of Sri Lanka Medical Association.

\section{Results}

\section{General Information}

Among a total of 104 intern medical officers(IMO) who have started their internship on 10.12.2012 in Castle street hospital for women(CSHW), De Soysa maternity hospital(DMH), Lady Ridgway hospital(LRH) and the National hospital of Sri Lanka(NHSL), $100(96.1 \%)$ participated for the study completing the survey questionnaire. Among those 100 participants majority were females (53\%). Furthermore majority $(45 \%)$ have been graduated from the faculty of medicine Colombo while $15 \%$ were from faculty of medicine Ragama. At the time of data time of data collection all the participants were in their second appointment. A majority (24\%) were currently attached to surgical wards while $23 \%$ 
were in medical wards (Chart 2).However from the total $27 \%$ have completed 6 months training in Medicine , 24\% have completed Gyn \& Obs, 16\% have completed paediatrics while $8 \%$ have completed paediatric surgery.

\section{Practices of requesting HIV testing}

57 participants $(\mathrm{N}=100)$ have decided at least once on their own to order an HIV test in the ward setting while 43 have never ordered an HIV test for the last 6 months. However only 54 participants could remember the approximate number of HIV tests that they have ordered on their own. Therefore these 54 participants have performed a total of about 182 tests with a minimum of 1 and a maximum of 12 (mean 3.37).

However, totally $85 \%$ of the participants have ordered HIV testing at least after senior doctors' opinion in the unit including the consultants. As instructed by the seniors they have ordered a total of 584 tests with a maximum of 53 times and a minimum of 2(mean 12.29). Therefore totally about $766 \mathrm{HIV}$ tests have been offered to the patients who got inward treatment for various illnesses. Among the total number HIV tests requested by the intern medical officers, majority ( $72 \%$ ) have requested considering the patients clinical findings. However $17 \%$ of the occasions HIV testing has been requested based on the patients real or perceived high risk behaviours. $6 \%$ of the testing has been done prior to invasive procedures as a prerequisite for the procedure while $5 \%$ has been done following needle prick injuries to the health care workers. The overall reasons for HIV testing are summarized in figure 1.

Among the problems encountered by the IMOs related to HIV testing, majority $(26.6 \%)$ had problems due report delay to while $22.2 \%$ had problems in explaining the relevance of the test to the patient in getting the consent .

\section{Knowledge on HIV testing indications}

Among the 100 participants whose knowledge was assessed by using the standard HIV testing indications recommended by the British HIV association, 99\% believed that the HIV testing should be offered for people who are diagnosed or suspected to have any sexually transmitted infection. $91 \%$ knew that it should be done in evaluation of pyrexia of unknown origin. All the indications and the responses are shown in figure 2. Among the recommended indications for HIV testing responses of the participants further analyzed according to the clinical conditions of WHO staging. Therefore responses obtained for WHO HIV stages separately illustrated in figures 3,4 and 5 .

The knowledge on the indications of HIV testing was graded according to the number of correct answers made by the respondents. Therefore 2 marks offered for each correct response and totally there were 50 items to assess the knowledge on indications. Thereby the maximum mark which a respondent could obtain was 100 . According to the total marks that the respondent has obtained they were categorized in to three groups. If the individual has scored more than 75 marks the knowledge was categorized as 'good'. If the total marks are in between 50- 74, they were included in the 'average' group and when the total marks are less than 49 it was categorized as 'poor'. Thereby the knowledge of 51 out of 100 respondents was 'average' while 45 had 'poor' knowledge. There were only 4 with good knowledge.

\section{Knowledge on testing procedure}

$81 \%$ of the respondents knew the correct specimen which should be sent for HIV testing. Regarding the knowledge on the container used for specimen collection, $88 \%$ were able to give the correct answer. However only $24 \%$ of the respondents knew the required blood volume for HIV testing and only $69 \%$ knew the correct venue where it is done.

Only $63 \%$ knew that all the positive HIV screening testing should be confirmed with a second confirmatory test. $75 \%$ believed that they should where double gloves when blood is drawn for HIV testing. $31 \%$ believed that the HIV testing can be requested as any other routine blood test without having any prerequisites while $11 \%$ were totally unaware whether there are prerequisites or not. 


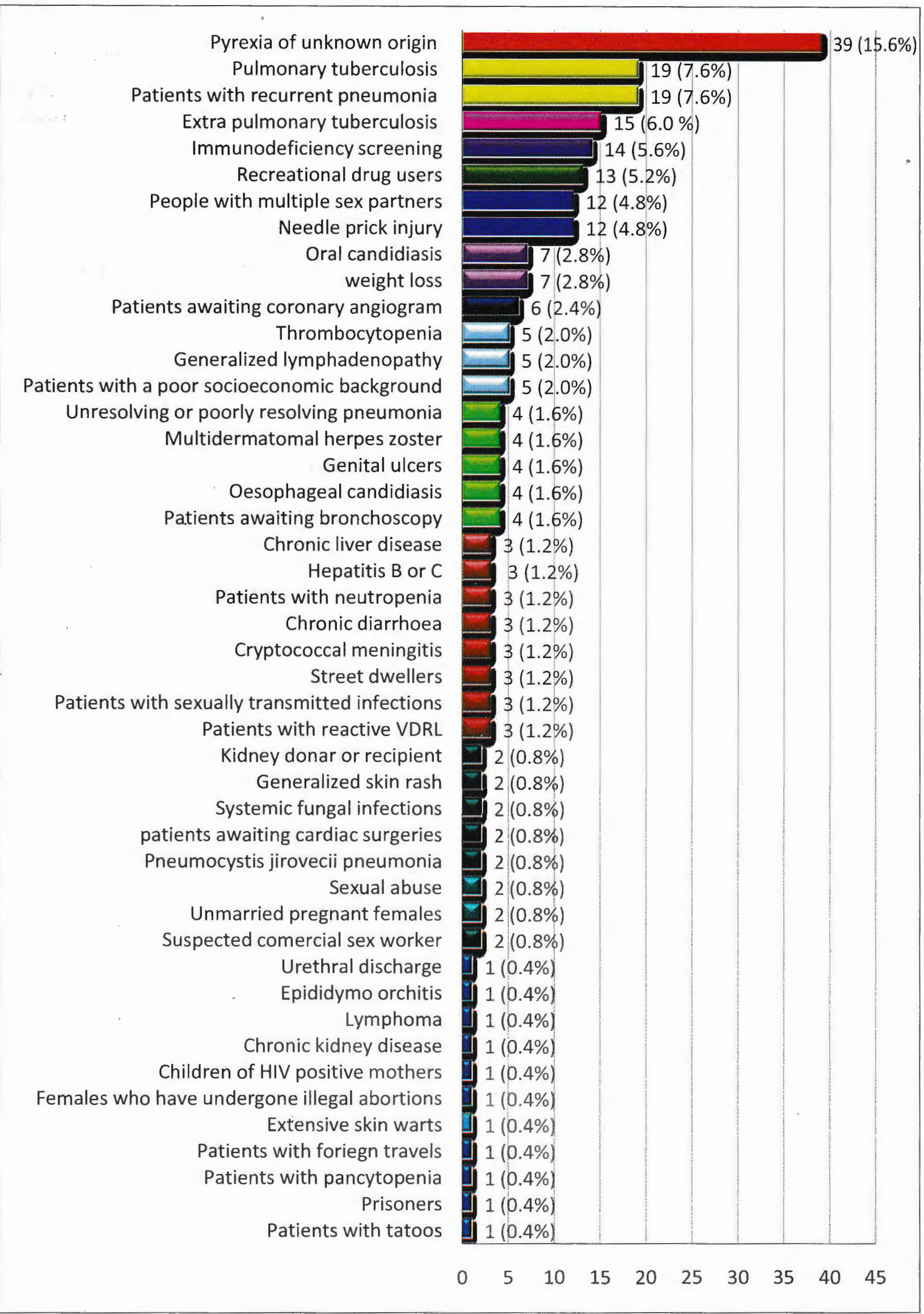

Figure 1: Number of HIV testing requested for different scenarios. 


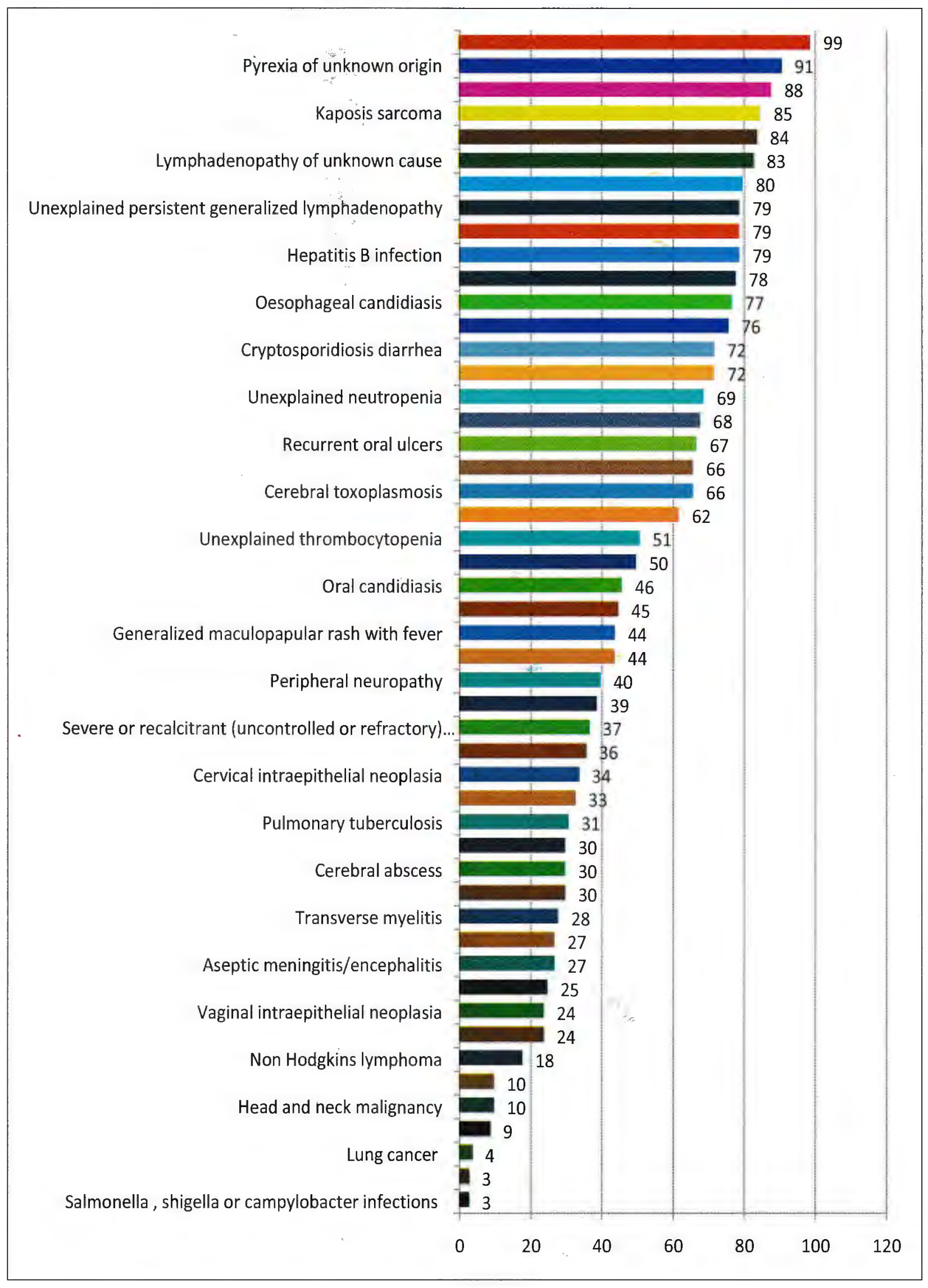

Figure 2: Knowledge on HIV testing indications. 


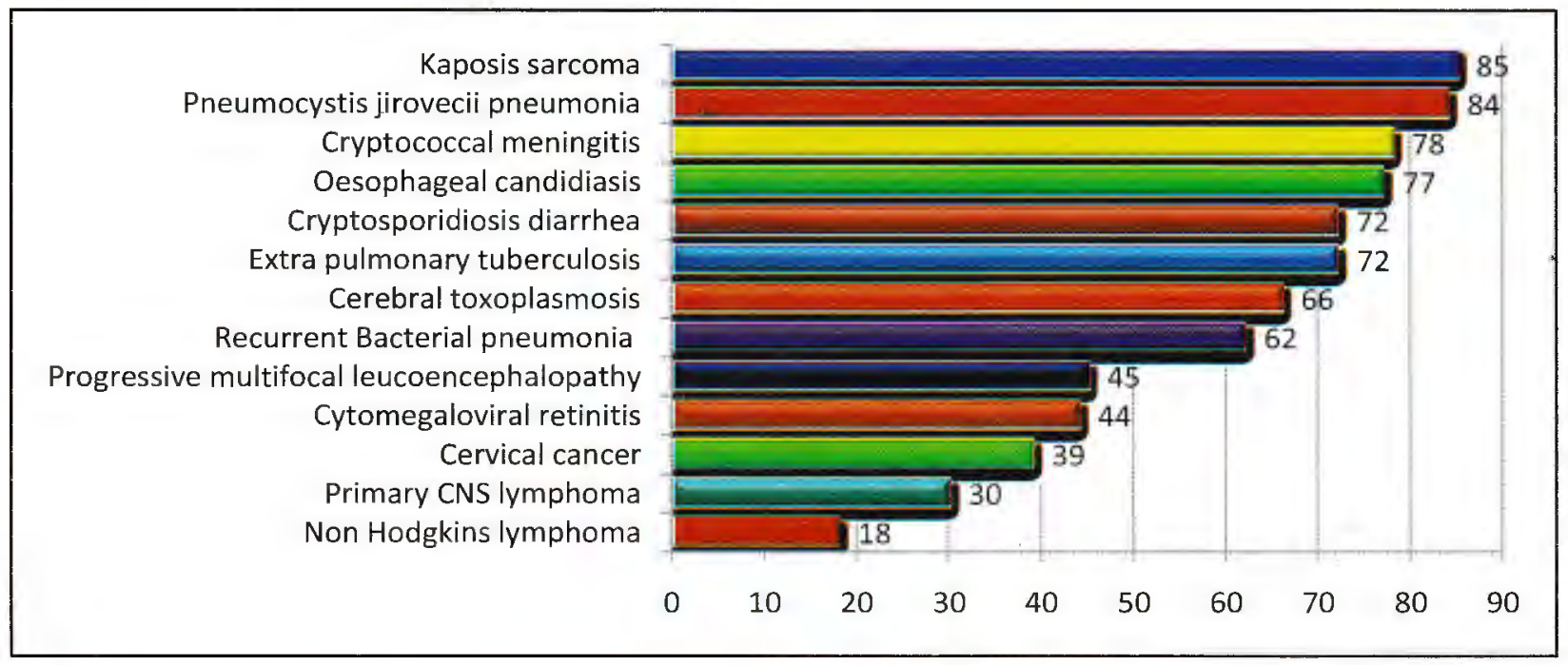

Figure 3: Knowledge on WHO stage 4 conditions.

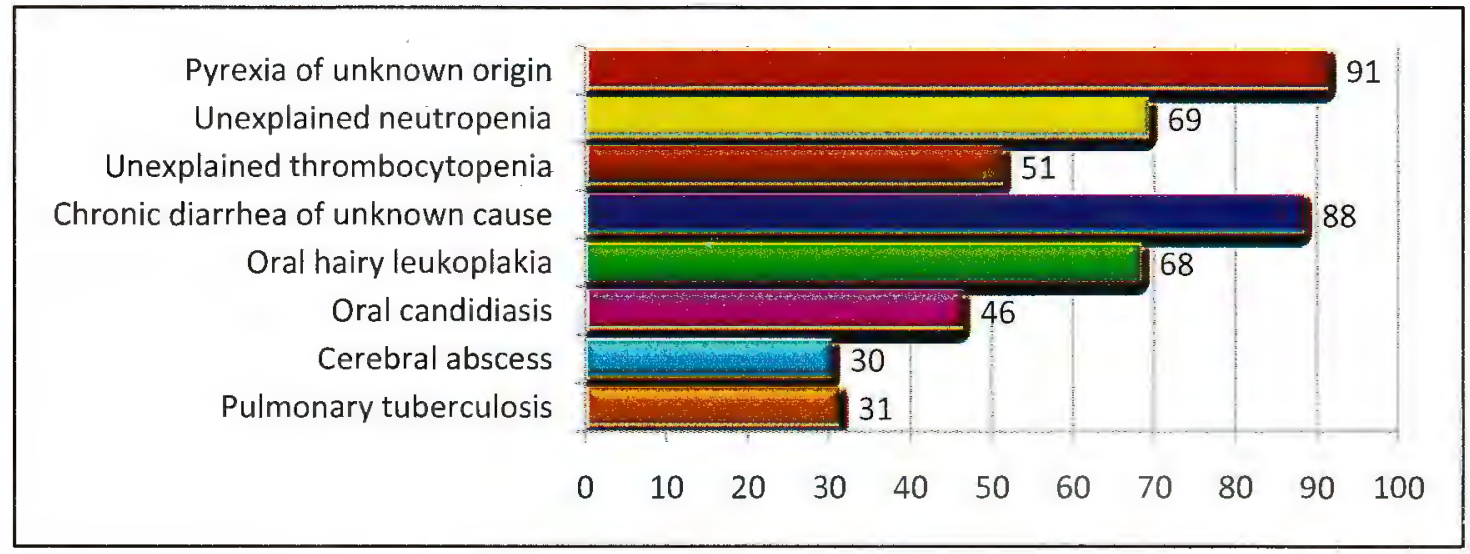

Figure 4: Knowledge on WHO stage 3 conditions.

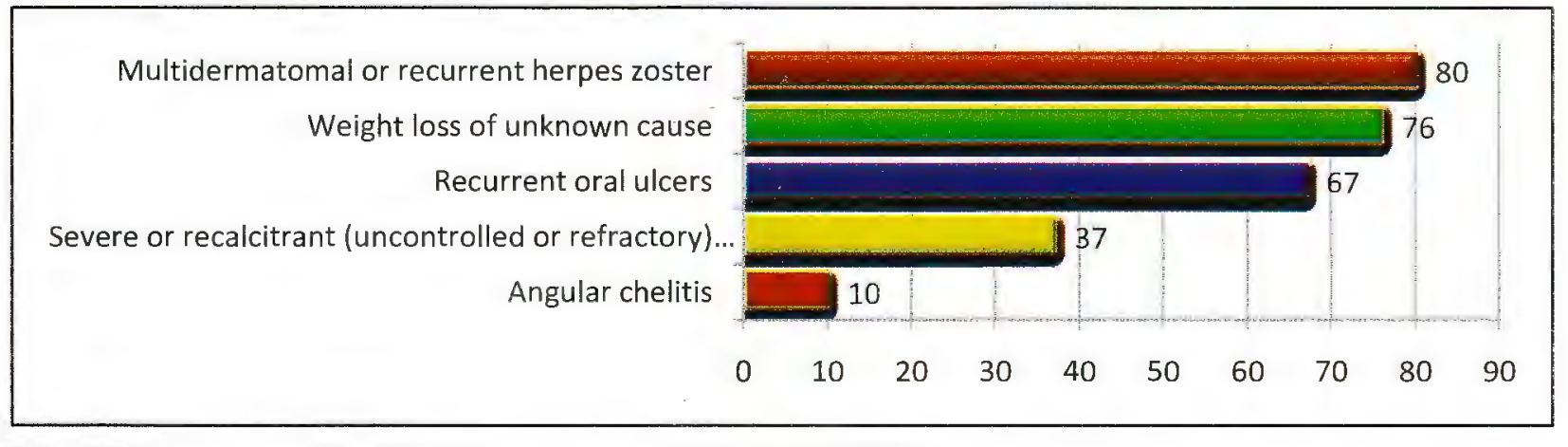

Figure 5: Knowledge on WHO stage 2 conditions.

The knowledge on the indications of HIV testing was graded according to the number of correct answers made by the respondents. Therefore 2 marks offered for each correct response and totally there were 50 items to assess the knowledge on indications. Thereby the maximum mark which a respondent could obtain was 100 . According to the total marks that the respondent has obtained they were categorized in to three groups. If the individual has scored more than 75 marks the knowledge was categorized as 'good'. If the total marks are in between 50-74, they were included in the 'average' group and when the total marks are less than 49 it was categorized as 'poor'. Thereby the knowledge 
significant problem for the IMOs in obtaining the consent and certain barriers seems to be existing in report tracing.

\section{Recommendations}

Reducing the high number of late diagnoses is a clinical and public health priority. To achieve this, it is recommended to set clinical guidelines for HIV testing in ward setting and policy dispersal along with the display of posters mentioning the clinical indications of HIV. CME/in service training of health care providers to upgrade the knowledge on HIV testing. Furthermore to explore the possibilities to expand the content related to HIV testing in the medical curriculum.

\section{Acknowledgements}

We thank Dr C.D Wickramasuriya (Consultant Venereologist, National STD/AIDS Control

Programme.) for her valuable guidance.

\section{Conflict of interest}

No conflict of interest declared by authors

\section{Funding}

No funding assistance

\section{References}

1.National STD/AIDS control programme of Sri Lanka. HIV quarterly update reports (WWW) NSACP.Available from: $\underline{h t t p: / /}$ www.aidscontrol.gov.lk/web/ index.php?option $=$ com_content\&view $=$ article\&id $=154 \&$ Itemid $=123$ \&lang $=$ en

2. BHIVA, BASHH, BIS. UK National Guidelines for HIV Testing. 2008. http://www.bhiva.org/ûles/ ûle1031097.pdf (accessed 29 Dec 2010).

3. Country progress report Sri Lanka (2010-2011), 2012.Availablefrom:http:// aidsreportingtool.unaids.org/116/sri-lanka-reportncpi.
4. WHO, Guidance on provider initiated HIV testing and counseling in health facilities, 2007, ISBN 978 9241595568 .

5. Smith RD, Delpech VC, Brown AE, et al. HIV transmission and high rates of late diagnoses among adults aged 50 years and over. AIDS 2010; 24:2109e15.

6. Guideline for intern medical officers 2012, Ministry of health. 